\title{
Robust Visual Homing with Landmark Angles
}

\author{
John $\operatorname{Lim}^{1,2}$ \\ ${ }^{1}$ Department of Information Engineering, RSISE \\ Australian National University \\ john.lim@rsise.anu.edu.au
}

\author{
Nick Barnes ${ }^{1,2}$ \\ ${ }^{2}$ Canberra Research Laboratory, \\ NICTA, Australia \\ nick.barnes@nicta.com.au
}

\begin{abstract}
This paper presents a novel approach to visual homing for robot navigation on the ground plane, using only the angles of landmark points. We focus on a robust approach, leading to successful homing even in real, dynamic environments where significant numbers of landmark points are wrong or missing. Three homing algorithms are presented, two are shown to be provably convergent, and the other shown to converge empirically. Results from simulations under noise and robot homing in real environments are provided.
\end{abstract}

\section{INTRODUCTION}

Visual homing is the problem of using information from visual images to return to some goal or home location after an agent has been displaced from that initial location.

Many approaches are possible. If some metric map of the environment was constructed (eg: using laser, sonar, stereo within a SLAM framework [1,2]), the agent can plan its return path using the map. Alternatively, the estimated camera motion from two views can give the homing direction using computer vision algorithms such as the 8-point algorithm [3]. Visual servoing [4] and other approaches [5, 6] are also possible.

Here, we focus on local visual homing methods which are typically used within graph-like topological maps $[7,8$, 9] where connected nodes represent nearby locations in the environment. A local homing algorithm is then used to drive the agent from one node to the next. Various local homing methods exist, including [10,11, 12, 13, 14, 15, 16, 17] and many others, which can be found in reviews such as [18]. The elegance and simplicity of these methods make them attractive not only for robotics, but also for modeling homing behavior in insects. (Indeed, many of these algorithms were directly inspired by or aimed at explaining insect homing.)

Using only the angles of landmark or feature points, we follow in the footsteps of other biologically inspired homing methods. These include the snapshot model [16], which matches sectors of the image snapshot taken at the goal position with the sectors in the current image to obtain 'tangential' and 'radial' vectors used to perform homing. The average landmark vector method [11] computes the average of several unit landmark vectors (a vector pointing from the agent to some landmark point) and obtains a homing vector by subtracting the average landmark vectors at the current and goal positions. The average displacement model of [15] and the work of [19] are examples of other such methods. These rely on some globally known compass direction in order to perform a correct comparison of landmark vectors observed at the current and goal positions. More recently, $[10,20]$ proposed a compass-free framework which works for any combination of start, goal and landmark positions on the entire plane. This approach uses a 'basic' and a 'complementary' control law that performs homing with three or more landmarks.

In our work, we assume that correspondences have been found between the landmark points in an image taken at the current position with those in an image taken at the goal location. The robot starts at some point, calculates the homing direction and moves in that direction. This is iterated and the robot moves step by step, until it arrives at the goal location. The landmark points used are image features obtained, matched or tracked with methods such as Scale Invariant Feature Transform (SIFT) matching [22], Harris corners [23], KLT [24] and others. These are 'naturally' occurring landmark points, rather than objects placed in the scene for the sole purpose of being an easily tracked and unmoving landmark. While we focus on local, short-range homing, this can be integrated with a topological map framework [7, 8, 9, 20] to perform long-range homing and localization tasks.

Section II introduces three algorithms. Firstly, a homing algorithm with both the robot and landmark points lying on the same plane is presented and shown to converge theoretically. Next, we investigate the case of planar homing with general, 3D landmark points and present two algorithms - the conservative method, which has theoretically guaranteed convergence and a non-conservative method which is demonstrated to converge empirically. Implementation details are in Section III and experimental results are in Section IV.

Motivation. We aim to address the issues of:

Robustness to outliers. In general, existing methods assume that a correct correspondence has been found between landmarks seen in the goal and current positions. However, if some of these landmarks have moved, are moving, were occluded, or were erroneously matched, there is often no provision to ensure successful homing. We term such moved or mismatched landmarks as outliers in the observed data. We propose a voting framework that efficiently integrates information from all landmarks to obtain homing cues that are extremely robust to outliers and other types of measurement noise.

Provable convergence. Homing is not provably successful in most of the cited local homing methods. The work of [10] showed successful homing through extensive simulations. [12] provided the first planar homing algorithm with a proof 
of convergence. This paper differentiates itself from [12], by proposing provably convergent algorithms for the more general case where the relative rotation between current and goal positions need not be known (compass-free), and where the landmarks need not lie on the same plane as the robot.

\section{THEORY}

Let the goal or home be a point $G$ and the current location of the robot be the point $C$. Images of the environment are taken at both points. At each step, the robot uses this image pair to compute and move in some homing direction, h. Hence, $C$ changes location with each iteration and homing is successful when $C$ converges on $G$. Define convergence as moving the robot to within some neighbourhood of $G$ and having it remain in that neighbourhood. We begin with the observation that:

Observation 1: The robot converges, if at each iteration, it takes a small step in the computed direction $\mathbf{h}$, such that the angle between $\overrightarrow{C G}$ and $\mathbf{h}$ is less than $90^{\circ}$.

$C$ lies on a circle centered on $G$ with radius $|\overrightarrow{C G}|$. If the movement of the robot is small, it will always move into the circle if homing direction $\mathbf{h}$ deviates from $\overrightarrow{C G}$ by less than $90^{\circ}$ (At exactly $90^{\circ}$ from $\overrightarrow{C G}$, the robot moves off the circle in a tangent direction). This ensures that each step the robot makes will take it a little closer to the goal (since it is moving into the circle). Hence the robot-goal distance decreases monotonically, and at the limit, it will reach $G$. (In practice, robot motion is not infinitesimally small, but as long as the angle between $\mathbf{h}$ and $\overrightarrow{C G}$ is not too close to $90^{\circ}$, the above still holds true.)

\section{A. The Case of the Planar World}

Consider the case in which both robot and landmark points lie on a plane. Given 2 landmarks $L_{1}$ and $L_{2}$ (Figure 1(a)), and the current position at point $C$, the inter-landmark angle is the angle between the two rays $\overrightarrow{C L_{1}}$ and $\overrightarrow{C L_{2}}$. All points on circular arc $L_{1} C L_{2}$ observe the same angle $\angle L_{1} C L_{2}$. Following [10], we define a convention where angle $\angle L_{1} C L_{2}$ is consistently measured, going from $\overrightarrow{C L_{1}}$ to $\overrightarrow{C L_{2}}$, in a anticlockwise (or clockwise) direction. Then any point on arc $L_{1} C L_{2}$ will have acute $\angle L_{1} C L_{2}$ (or obtuse, if measuring in the clockwise direction). The inter-landmark angle observed at the current position, $\angle L_{1} C L_{2}$, is the current angle, whilst that observed at the goal position, $\angle L_{1} G L_{2}$, is the goal angle.

The circular arc $L_{1} C L_{2}$ is termed a horopter. The dashed landmark line $L_{1} L_{2}$ splits the plane into upper and lower halfplanes. Let the set of points on the horopter be $R_{0}$; let the region in the upper half-plane and within the horopter be $R_{1}$; the region outside the horopter (shaded region in Figure 1(a)) be $R_{2}$; and the region in the lower half-plane be $R_{3}$.

In the configuration of Figure 1(a) and using the anticlockwise angle measurement convention, if $C$ lies on the horopter, and $G$ lies within $R_{1}$, that is $G \in R_{1}$, then $\angle L_{1} G L_{2}>$ $\angle L_{1} C L_{2}$. However, if $G \in R_{2}$, then $\angle L_{1} G L_{2}<\angle L_{1} C L_{2}$. Furthermore, the anticlockwise convention for measuring angles implies that if point $P \in R_{1} \cup R_{2}$, then $\angle L_{1} P L_{2}$ is acute, but if $P \in R_{3}$, then $\angle L_{1} P L_{2}$ is obtuse. Therefore,
$C \in R_{0} \cup R_{1} \cup R_{2}$ and $G \in R_{3}$, implies $\angle L_{1} C L_{2}<\angle L_{1} G L_{2}$ since acute angles are always smaller than obtuse ones.

The acute-obtuse cases are reversed if $C$ lies on the other side of the line $L_{1} L_{2}$ (the lower half-plane). Angle $\angle L_{1} C L_{2}$ is now acute when measured in the clockwise direction and obtuse if measured in the anticlockwise. An analysis will yield relationships symmetrical to the above.

With this, given knowledge of whether the current or the goal angle is larger, one can constrain the location of $G$ to one of regions $R_{1}, R_{2}, R_{3}$. However, since distance from the current point to the landmarks is unknown, we know neither the structure of the horopter nor that of the line $L_{1} L_{2}$. Even so, one can still obtain valid but weaker constraints on the location of $G$ purely from the directions of the landmark rays.

These constraints on $G$ come in two types. Type 1: Define a region $R_{A 1}$ that lies between vectors $\overrightarrow{C L_{1}}$ and $\overrightarrow{C L_{2}}$ (shaded region in Figure 1(b)). Let the complement of $R_{A 1}$ be $R_{B 1}=$ $R_{A 1}^{c}=\Pi \backslash R_{A 1}$, where $\Pi$ is the entire plane and $\backslash$ denotes set difference. $R_{B 1}$ is the Type 1 constraint region (the entire unshaded region in Figure 1(b)) and $G$ must lie within $\underline{R_{B 1}}$.

Type 2: Let $R_{A 2}$ lie between the vectors $-\overrightarrow{C L_{1}}$ and $-\overrightarrow{C L_{2}}$ (shaded region in Figure 1(c)). The Type 2 constraint region is the complement set, $R_{B 2}=R_{A 2}^{c}$ (the unshaded region).

Lemma 1: If an acute current angle is greater than the goal angle, then $G \in R_{B 1}$ but if it is less than the goal angle, then $G \in R_{B 2}$. If an obtuse current angle is greater than the goal angle, then $G \in R_{B 2}$ but if it is less, then $G \in R_{B 1}$.

Proof: If $\angle L_{1} C L_{2}$ is acute and $\angle L_{1} C L_{2}>\angle L_{1} G L_{2}$, the goal, $G$, must lie in $R_{2} . R_{A 1}$ is the shaded area in Figure 1(b), which does not intersect $R_{2}$. One can see that for any $C$ lying on the horopter, $R_{A 1}$ never intersects $R_{2}$. $R_{2}$ is a subset of $R_{B 1}$. Hence, $G \in R_{2} \Rightarrow G \in R_{B 1}$.

Conversely, if $\angle L_{1} C L_{2}$ is acute and $\angle L_{1} C L_{2}<\angle L_{1} G L_{2}$, then $G \in R_{1} \cup R_{3}$ and $G \notin R_{2} . R_{A 2}$ is the shaded area in Figure 1(c) and $R_{A 2} \cap\left(R_{1} \cup R_{3}\right)=\emptyset$ for any point $C$ on the horopter. So, $G \in\left(R_{1} \cup R_{3}\right) \subset R_{B 2}$.

For obtuse $\angle L_{1} C L_{2}$ (when using the clockwise convention), the proof is symmetrical to the above (that is, if $\angle L_{1} C L_{2}>\angle L_{1} G L_{2}$, then $G \in R_{1} \cup R_{3}$ and $\left(R_{1} \cup R_{3}\right) \subset$ $R_{B 2}$ so $G \in R_{B 2}$. Also, if $\angle L_{1} C L_{2}<\angle L_{1} G L_{2}$, then $G \in R_{2}$ and $R_{2} \subset R_{B 1}$ so $G \in R_{B 1}$ ).

From the constraints on $G$, we can now define constraints on $\overrightarrow{C G}$. Some region, $R$, consists of a set of points, $\left\{P_{1}, P_{2} \cdots P_{i}, \cdots\right\}$. We define a set of direction vectors:

$$
D=\left\{\mathbf{d}_{\mathbf{j}} \mid \mathbf{d}_{\mathbf{j}}=\frac{\overrightarrow{C P_{i}}}{\left|\overrightarrow{C P_{i}}\right|}, \forall i>0, j \leqslant i\right\}
$$

such that for every point in set $R$ there exists some $k>0$ and some $\mathbf{d}_{\mathbf{j}} \in D$ such that $P_{i}=C+k \mathbf{d}_{\mathbf{j}}$. (A robot starting at $C$ and moving $k$ units in direction $\mathbf{d}_{\mathbf{j}}$ will arrive at $\underset{P_{i}}{\longrightarrow}$ ). As an example, consider the case in Figure 1(b). Let $\overrightarrow{C L_{1}}$ correspond to the polar coordinate angle, $\theta$, of $\theta \overrightarrow{C L_{1}}=0$, and let the direction of $\theta$ vary in the anticlockwise direction. Then, region $R_{A 1}$ maps to the fan of vectors with polar angle $0<\theta<\theta_{\overrightarrow{C L_{2}}}$ whilst region $R_{B 1}$ maps to the vectors with polar angle $\theta_{\overrightarrow{C L_{2}}}<\theta<360^{\circ}$. 


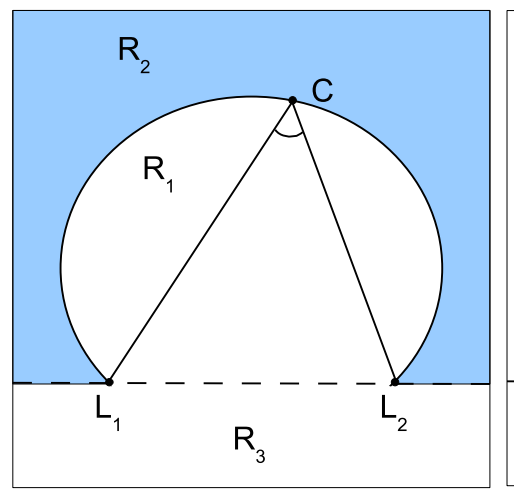

(a)

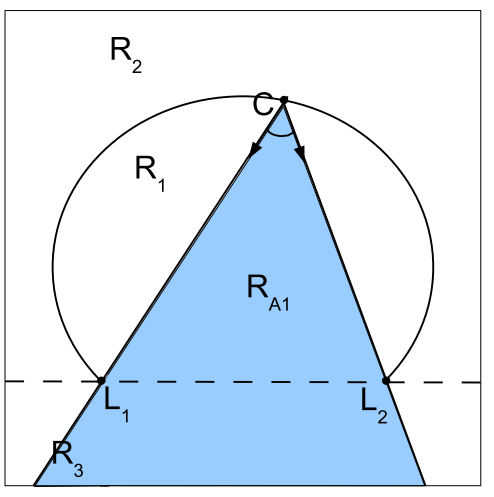

(b)

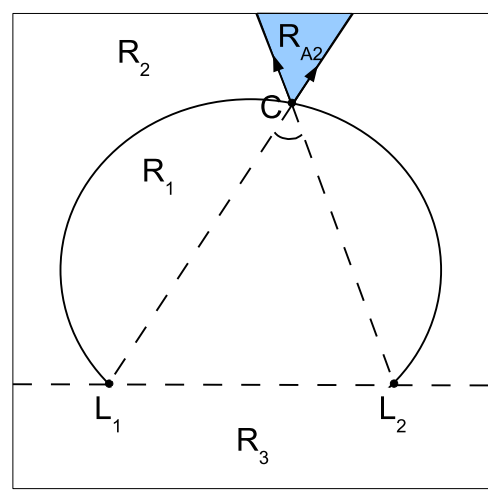

(c)

Fig. 1. (a) Horopter $L_{1} C L_{2}$ and line $L_{1} L_{2}$ splits the plane into 3 regions. (b-c) Regions $R_{A 1, A 2}$ (shaded) and $R_{B 1, B 2}$ (everything that is unshaded).

$\overrightarrow{C G}$ is the vector pointing from current to goal location. Recovering it guarantees convergence (moving in the direction $\overrightarrow{C G}$ repeatedly will, inevitably, bring the robot to $G$ ). It also gives the most efficient path (the straight line) to the goal. In the following, we are interested only in the direction of $\overrightarrow{C G}$ and we will use $\overrightarrow{C G}$, and the unit vector in the direction $\overrightarrow{C G}$, interchangeably. Then:

Theorem 1: From one landmark pair, Lemma 1 constrains $G$ within regions $R_{B 1}$ or $R_{B 2}$. Equation 1 maps this to a set of directions, $D$, where $\overrightarrow{C G} \in D$. For $N$ landmarks, we have up to ${ }^{N} C_{2}$ vector sets $\left\{D_{1}, D_{2}, \cdots D_{N^{N}}\right\} . \overrightarrow{C G}$ lies in their intersection, $D_{\text {res }}=D_{1} \cap D_{2} \cap \cdots \cap D_{N_{N} C_{2}}$.

Assuming isotropically distributed landmarks, as $N \rightarrow \infty$, $D_{\text {res }}=\overrightarrow{C G}$. In practice, successful homing only requires $N$ to be large enough, such that a homing direction can be chosen, that is less than $90^{\circ}$ from every vector in $D_{\text {res. }}$. It will then be less than $90^{\circ}$ from $\overrightarrow{C G}$ and from Observation 1, the robot will converge. A stricter condition is to have the maximum angle between any two vectors in $D_{\text {res }}$ less than $90^{\circ}$. Then all vectors in $D_{\text {res }}$ will be less than $90^{\circ}$ from $\overrightarrow{C G}$.

\section{B. Robot on the Plane and Landmarks in $3 D$}

Whilst the robot can move on some plane with normal vector, $\mathbf{n}$, the observed landmark points will, in general, not lie on that plane. Here, we extend the previous results to this more general situation. It is not unreasonable to assume that the robot knows which direction is 'up', that is, the normal to the ground plane. Then, we can measure angles according to the same clockwise or anticlockwise conventions as before.

Let $C, G$ lie on the x-y plane (so normal vector $\mathbf{n}$ is the z-axis) and suppose $C$ lies in the negative-x region. There always exists some plane passing through two 3D points such that this plane is orthogonal to the $\mathrm{x}-\mathrm{y}$ plane. So, without loss of generality, we can let the landmark pair $L_{1}, L_{2}$ lie on the y$\mathrm{z}$ plane. There exists a circular arc passing through $L_{1}, C$ and $L_{2}$. Revolving the arc about line $L_{1} L_{2}$ sweeps out a surface of revolution. The portion of the surface that lies in the negative- $x$ region of $\Re^{3}$ is the horopter surface. All points on the horopter have inter-landmark angles equal to $\angle L_{1} C L_{2}$.
The intersection of this 3D horopter surface with the $\mathrm{x}$ y plane containing $C$ and $G$ is a 2D horopter curve (some examples shown in Figure $2(\mathrm{a}-\mathrm{f})$ ). Rather than a circular arc, the curve is elliptical. Let region $R_{1}$ be the set of all points inside the horopter curve and in the negative-x region; let $R_{2}$ be the set of points in the negative-x region and outside the horopter; let $R_{3}$ be the set of points in the positive-x region. Then, the inequality relationships between $\angle L_{1} C L_{2}$ and $\angle L_{1} G L_{2}$ if $C$ lay on the horopter and $G$ in one of regions $R_{1}, R_{2}$ or $R_{3}$, are similar to those discussed in Section II-A.

Since landmark distances are unknown, once again, we attempt to constrain $G$ using only landmark directions. The difference is that we now use the projected landmark vectors $\overrightarrow{C \overrightarrow{L_{1}}}$ and $\overrightarrow{C \tilde{L}_{2}}$ instead of the landmark vectors as was previously the case. The projection of a landmark ray vector, $\overrightarrow{C L_{1}}$, onto the plane is $\overrightarrow{C \tilde{L_{1}}}=\mathbf{n} \times\left(\overrightarrow{C L_{1}} \times \mathbf{n}\right)$ where $\tilde{L_{1}}$ is the projection of $L_{1}$ onto the plane and $\times$ is the cross product.

The horopter curve intersects the $\mathrm{y}$-axis at $H_{1}, H_{2}$ and the projected landmark rays, $\overrightarrow{C \overrightarrow{L_{1}}}$ and $\overrightarrow{C \bar{L}_{2}}$ intersect the y-axis at $\tilde{L_{1}}, \tilde{L_{2}}$. However, whilst $L_{1}, L_{2}$ lie on the horopter, the projected landmark rays are such that $\tilde{L_{1}}, \tilde{L_{2}}$ do not lie on the horopter in general. (If they did, then the problem is reduced to that of Section II-A.) Let $P_{y}$ be the y-coordinate of point $P$. Three cases arise: (Case 1) both $\tilde{L_{1 y}}, \tilde{L_{2 y}}$ lie in the interval $\left[H_{1 y}, H_{2 y}\right]$; (Case 2 ) one of $\tilde{L_{1 y}}$ or $\tilde{L_{2 y}}$ lies outside that interval; (Case 3) both $\tilde{L_{1 y}}, \tilde{L_{2 y}}$ lie outside $\left[H_{1 y}, H_{2 y}\right]$.

For Case (1), the results of Lemma 1 hold except that the Type 1 and 2 constraint regions are now bounded by projected landmark rays. Figure 2(a) illustrates the case of $G \in R_{2}$. The shaded region is $R_{B 1}=R_{A 1}^{c}$, which is a Type 1 constraint bounded by $\overrightarrow{C \tilde{L_{1}}}, \overrightarrow{C \tilde{L_{2}}}$ such that $G \in R_{B 1}$ (since $R_{2} \subset R_{B 1}$ ) for any $C$ on the horopter curve. Meanwhile, Figure 2(b) illustrates the case of $G \in R_{1} \cup R_{3}$ and the shaded region is the Type 2 constraint region, $R_{B 2}=R_{A 2}^{c}$ bounded by $-\overrightarrow{C \tilde{L}_{1}},-\overrightarrow{C \tilde{L_{2}}}$ such that $G \in\left(R_{1} \cup R_{3}\right) \subset R_{B 2}$. Using Equation 1, these regions can be mapped to sets of possible homing directions.

Unfortunately, Lemma 1 does not always hold for Cases (2) 


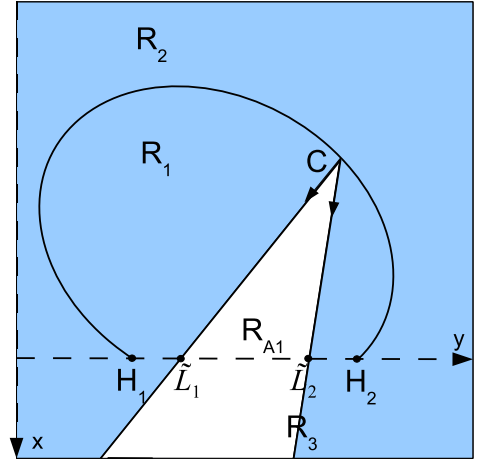

(a)

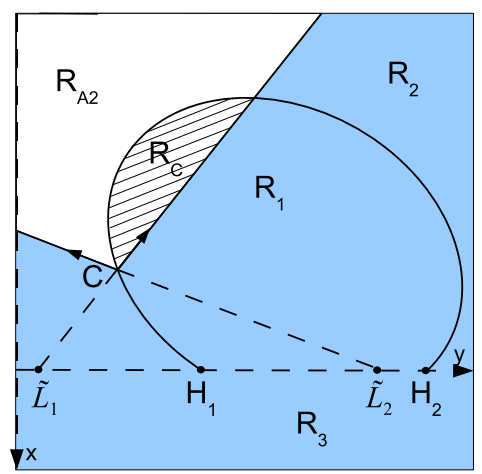

(d)

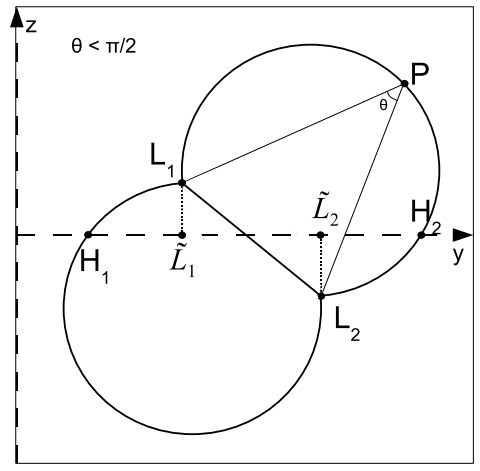

(g)

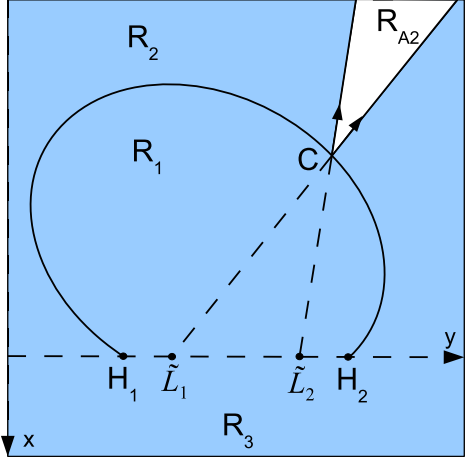

(b)

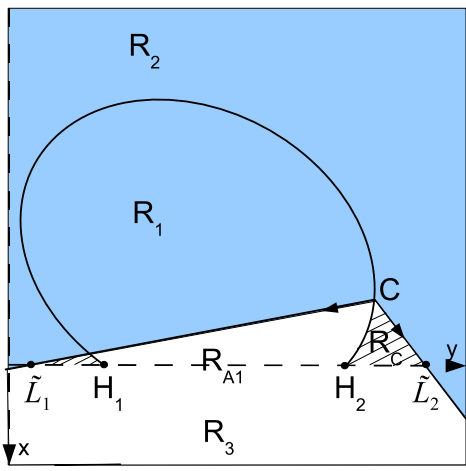

(e)

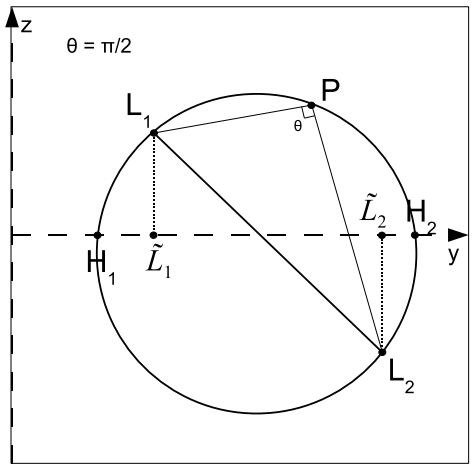

(h)

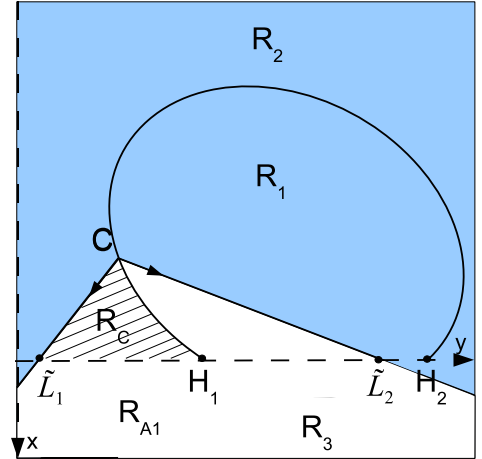

(c)

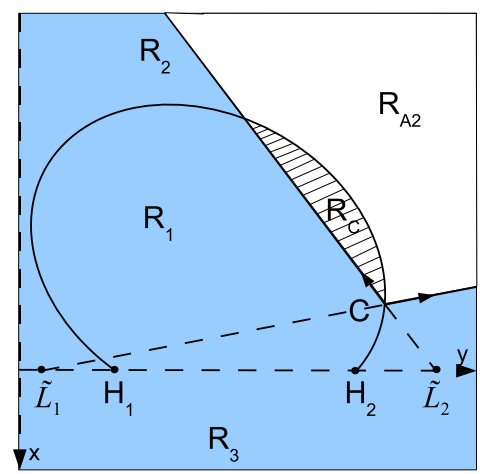

(f)

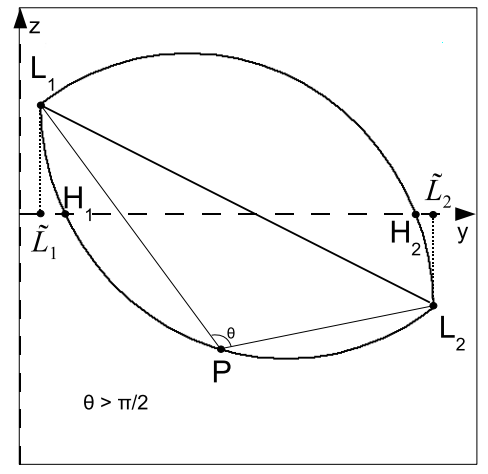

(i)

Fig. 2. Intersection of horopter with x-y plane. Regions $R_{B 1}, R_{B 2}$ constraining location of $G$ are shaded. The complement regions, $R_{A 1}, R_{A 2}$ are unshaded. (a-b) Case1: both $\tilde{L_{1 y}}, \tilde{L}_{2 y}$ in $\left[H_{1 y}, H_{2 y}\right]$. (c-d) Case 2 : one of $\tilde{L_{1 y}}$ or $\tilde{L}_{2 y}$ outside $\left[H_{1 y}, H_{2 y}\right]$. (e-f) Case 3 : both $L_{1 y}, L_{2 y}$ outside $\left[H_{1 y}, H_{2 y}\right]$. (g-i) Intersection of horopter surface with y-z plane. 3 cases arising from $\theta<90^{\circ}, \theta=90^{\circ}$ and $\theta>90^{\circ}$.

and (3) such as in the example configurations in Figures 2(c, d) for Case (2) and (e, f) for Case (3). Regions $R_{C}$ are marked with diagonal hatching. In Figure 2(c), the Type 1 constraint, $R_{B 1}$ should contain $R_{2}$. However, $R_{C} \subset R_{2}$ but $R_{C} \not \subset R_{B 1}$. Likewise, in Figure 2(d), the Type 2 constraint, $R_{B 2}$ should contain $R_{1} \cup R_{3}$ but it misses out on $R_{C} \subset R_{1}$. This means that if $G$ lay in $R_{C}$, there would exist configurations of $L_{1}, L_{2}, C$ where $G$ would not lie in the constraint region.

Two approaches are possible - the first is a non-conservative approach that uses all constraints arising from all landmark pairs. This method will very probably converge and in all (thousands of) experiments (Section IV), was indeed observed to converge. A second approach uses only a subset of all the constraints and its convergence is theoretically guaranteed.

\section{Non-conservative Method - Likely Convergence}

As a result of the above problem, Theorem 1 will no longer hold. $D_{\text {res }}$ was previously defined as the intersection of all sets of possible homing directions arising from all the landmark pairs. This may not exist, so we will instead define $\bar{D}_{\text {res }}$, which is the intersection of the largest number of such sets.

One observes that the size of $R_{C}$ tends to be small relative to the total region in which $G$ can lie. For example, in the 
case of $G \in R_{2}$ and $R_{B 1}$ should contain $R_{2}$ but misses out the $R_{C}$ regions (Figures $\left.2(\mathrm{c}, \mathrm{e})\right), R_{C}$ is largest when $C$ is at the highest point on the horopter and $\tilde{L_{1 y}}, \tilde{L_{2 y}}$ approach $\pm \infty$. Even then, $R_{C}$ is small compared to all of region $R_{2}$.

Therefore, the probability of $G \in R_{C}$ is actually rather small. Assuming $N$ randomly scattered landmarks, out of ${ }^{N} C_{2}$ constraints, some proportion of constraints would give rise to these 'missed' regions, $R_{C}$, where there is potential for error. Of these, an even smaller number will actually be erroneous constraints, i.e. $G \in R_{C}$. These erroneous constraints map to a set of direction vectors that is 'wrong' in the sense that $\overrightarrow{C G}$ is not in this set.

The direction $\overrightarrow{C G}$ might not lie in $\bar{D}_{\text {res }}$. However, there is a good chance that $\overrightarrow{C G}$ lies close to it, and in fact, there is a very high probability that $\overrightarrow{C G}$ is less than $90^{\circ}$ from a homing direction chosen from the set $\bar{D}_{\text {res }}$ (eg: the average of all directions in $\bar{D}_{\text {res }}$ ). In the highly unlikely event of a wrong estimate, that is, $\overrightarrow{C G}$ being greater than $90^{\circ}$ from the homing direction, the robot will make a step that takes it further away from the goal than it was at its last position.

However, this alone is insufficient to cause nonconvergence. The robot will move to a new position, leading to a change in the directions of the landmark rays, and a new estimate of the new homing direction is found. In order for the robot to not converge to $G$, it would have to obtain so many wrong estimates that it was going in the wrong direction most of the time. This requires a stacking of many improbable odds and indeed, in no experiment was non-convergence ever observed. Even so, there is a remote but finite chance of failure, which leads us to the next method.

\section{Conservative Method - Guaranteed Convergence}

Assume the camera is mounted some distance above the ground and the $x-y$ plane (we are working in the camera coordinate frame) is parallel to the ground. Homing motion is then restricted to this $x-y$ plane. As before, landmark pair $L_{1}, L_{2}$ lies on the $\mathrm{y}-\mathrm{z}$ plane and the horopter surface is the surface swept out by revolving the arc $L_{1} C L_{2}$ about the line $L_{1} L_{2}$, restricted to the half-space that has $\mathrm{x}$-coordinates with the same sign as the sign of $C_{x}$.

There is a strategy for picking landmark pairs so that $\tilde{L_{1 y}}, \tilde{L_{2 y}}$ lie in the interval $\left[H_{1 y}, H_{2 y}\right]$. This is the Case 1 configuration which is free of erroneous constraints:

Lemma 2: If a landmark pair, $L_{1}, L_{2}$ is chosen such that one lies above the $\mathrm{x}-\mathrm{y}$ plane and one lies below it, and such that $\theta=\cos ^{-1}\left(\overrightarrow{C L_{1}} \cdot \overrightarrow{C L_{2}}\right) \leqslant 90^{\circ}$, then $\tilde{L_{1 y}}, \tilde{L_{2 y}} \in\left[H_{1 y}, H_{2 y}\right]$.

Here, $\theta$ is always acute; it differs from the inter-landmark angle which can be acute or obtuse depending on how it is measured. The intersection of the horopter surface with the $y-z$ plane is as shown in Figures 2(g-i) which depict the three cases arising when the angle, $\theta$, between two landmark rays as observed at any point $C$ on the horopter, is greater than, equal to or less than $90^{\circ}$. With one landmark above and one below the x-y plane, the line segment lying between $L_{1}$ and $L_{2}$ intersects the y-axis. $\tilde{L}_{1}, \tilde{L_{2}}$ are the projections of $L_{1}$ and $L_{2}$ onto the x-y plane, whilst $H_{1}$ and $H_{2}$ are the intersections of the horopter with the plane.
If $\theta \leqslant 90^{\circ}$ (Figures $2(\mathrm{~g})$ and $(\mathrm{h})$ ), it can be shown that $\tilde{L_{1 y}}, \tilde{L_{2 y}} \in\left[H_{1 y}, H_{2 y}\right]$ is always true for any $L_{1}, L_{2}$ satisfying these conditions. However, if $\theta>90^{\circ}$, this is not always true, as the counterexample of Figure 2(i) demonstrates.

Using only landmark pairs that have $\theta \leqslant 90^{\circ}$ is a conservative method that ensures only correct constraints are used, in the noise-free case. However, most of the discarded constraints will in fact be correct, according to the earlier argument that the probability that $G \in R_{C}$ is low. (Note that if insufficient pairs of landmarks meet the conditions of Lemma 2, one can still use the earlier, non-conservative method to home.)

The conservative method ensures Theorem 1 holds and $\overrightarrow{C G} \in D_{\text {res }}$. Hence, if there are sufficient landmarks such that a vector $\mathbf{h}$ which is within $90^{\circ}$ of all direction vectors in $D_{\text {res }}$ can be found, then moving in the direction of $\mathbf{h}$ will bring the robot closer to the goal. If this condition is met at each step of the robot's estimate and move cycle, convergence on $G$ is guaranteed as per Observation 1 .

\section{Algorithms And Implementation}

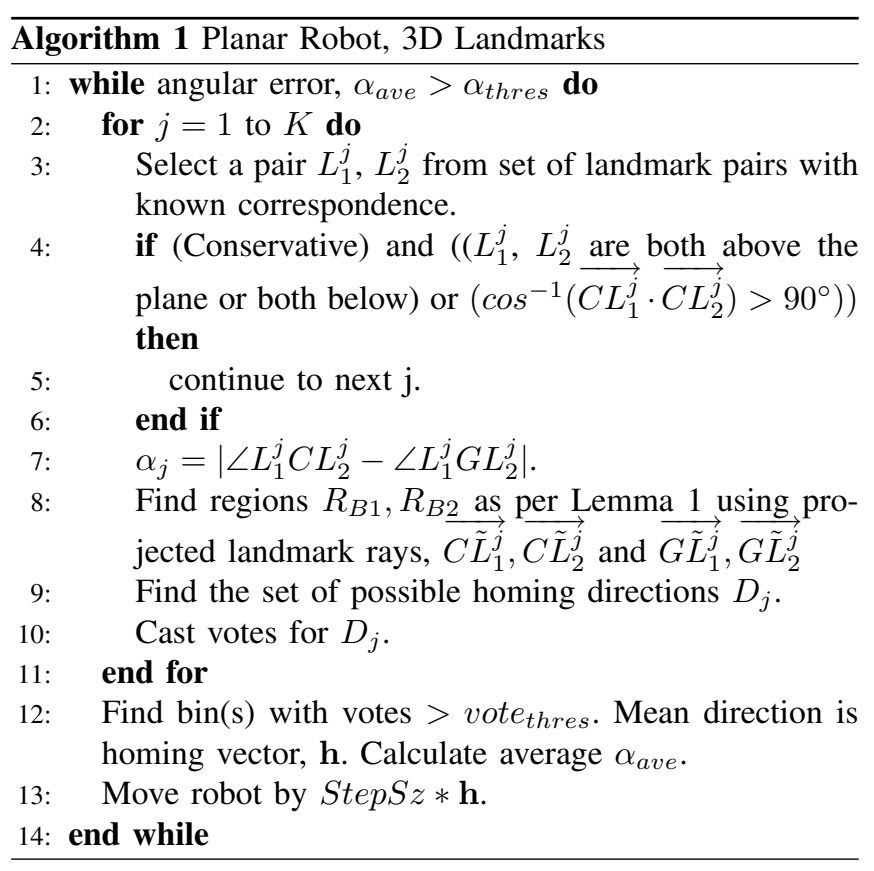

The homing approach proposed in Section II involves finding sets of possible homing directions and obtaining the intersection of all or of the largest number of these sets. A voting framework accomplishes this quickly and robustly. The table of votes divides the space of possible directions of movement into voting bins. For planar motion, voting is done in the range $\theta=\left[0,360^{\circ}\right)$. From the sets of possible homing directions $\left\{D_{1}, D_{2}, \cdots, D_{i}, \cdots\right\}$, votes for bins corresponding to the directions in each $D_{i}$ are incremented, and the bin(s) with maximum vote (or with votes exceeding a threshold, vote thres ) gives $D_{\text {res }}$ or $\bar{D}_{\text {res }}$. When more than one bin has maximum vote, we take the average direction to be the homing vector, $\mathbf{h}$. The non-conservative and conservative 
approaches for homing with planar robots and 3D landmarks are implemented in Algorithm 1.

In the algorithm, $K$ can be all ${ }^{N} C_{2}$ combinations of landmark pairs, or some random sample thereof. To sense whether the robot is far from or close to the goal, we average the inter-landmark angular error, $\alpha_{j}=\left|\angle L_{1}^{j} C L_{2}^{j}-\angle L_{1}^{j} G L_{2}^{j}\right|$ over all $j . \alpha_{\text {ave }}$ gives a measure of how similar the current and goal images are. Ideally, as the robot approaches the goal position, $\alpha_{\text {ave }} \rightarrow 0$. We specify that when $\alpha_{\text {ave }}<\alpha_{\text {thres }}$, homing is completed and the robot stops. $\alpha_{\text {ave }}$ may be used to control the size of robot motion, the variable StepSz.

Note that Algorithm 1 is more efficiently implemented by using the directions mapped from $R_{A 1}, R_{A 2}$ instead of $R_{B 1}, R_{B 2}$, since the former regions are typically smaller. This way, less computations (incrementing of votes) occur. $\mathbf{h}$, is then found from the minimum vote instead of the maximum.

\section{EXPERIMENTS AND RESULTS}

\section{A. Simulations}

In the Matlab simulations, landmarks were randomly scattered within a cube of $90 \times 90 \times 90$ units. The robot moves between randomly generated start and goal positions by iterating the homing behaviour. $\mathbf{h}$ was taken to be the average of the set of directions with maximum votes.

Firstly, for the noise-free case, 1000 trials were conducted for each of the planar conservative and planar non-conservative cases. Secondly, a series of experiments involving 100 trials each, investigated homing under outliers and Gaussian noise. Outliers were simulated by randomly replacing landmark rays with random vectors. The circle of possible directions of movement was divided into 360 voting bins ( $1^{\circ}$ per bin). We tested for up to $40 \%$ outliers and for Gaussian noise with standard deviation up to $9^{\circ}$.

The robot successfully homed in all experiments. This confirms the theoretical convergence proof for the conservative case, and gives empirical evidence supporting the statistical argument for likely convergence in the non-conservative case.

In order to examine the quality of homing, we compute the directional error (angle between $\overrightarrow{C G}$ and $\mathbf{h}$ ), which measures how much the homing direction deviates from the straight line home. The histograms in Figure 3(a-d) summarize the directional error in the noise and outlier experiments.

Recall, from Observation 1 that a homing step will take the robot closer to the goal if $\mathbf{h}$ is less than $90^{\circ}$ from $\overrightarrow{C G}$. For no noise and no outliers, all homing directions computed were indeed less than $90^{\circ}$ from $\overrightarrow{C G}$ (even for the non-conservative method). However, even with up to $40 \%$ of landmark rays being outliers, the number of homing estimates that were more than $90^{\circ}$ from $\overrightarrow{C G}$ was insignificant for the non-conservative case (Figure 3(b)), and less than $8 \%$ for the conservative case (Figure 3(a)). This means the robot was heading in the correct direction the vast majority of the time in spite of the outliers, hence homing was successful.

Successful convergence was observed in trials with Gaussian noise. Figures 3(c-d) illustrate the performance as the
Gaussian noise standard deviation is varied from $0^{\circ}$ to $9^{\circ}$. Performance degrades gracefully with noise but the proportion of homing vectors with directional error greater than $90^{\circ}$ was once again insufficient to prevent convergence.

It is interesting to note that the planar non-conservative case outperformed the planar conservative case. Voting proved particularly robust to the incorrect constraints arising in the non-conservative case. Since correct constraints dominate in number, these form a robust peak in the vote space which requires large numbers of incorrect constraints voting in consistency with each other, to perturb from its place. However, the incorrect constraints are quite random and do not generally vote to a consistent peak at all; and the 'missed' $R_{C}$ regions also tend to be small, so the total effect on the performance of the non-conservative case is quite minimal.

Conversely, although the conservative case guarantees convergence, that guarantee comes at the cost of throwing away all constraints that are not certain to be correct. In the process, many perfectly fine constraints are discarded as well. Thus, the set of directions with maximum votes was larger, and the average direction (taken as the homing direction) deviated further from $\overrightarrow{C G}$, compared to the non-conservative method.

\section{B. Real experiments}

Grid trials: A camera captured omnidirectional images of some environment at every $10 \mathrm{~cm}$ in a $1 \mathrm{~m} \mathrm{x} 1 \mathrm{~m}$ grid. With some point on the grid as the home position, the homing direction was calculated at every other point on the grid. The result is summarized in a vector-field representation where each vector is the direction a robot would move in if it was at that point in the field. By taking each point on the grid in turn to be the home position, we can examine how the algorithm performs in that environment for all combinations of start and goal positions (marked with a ' + ') within the grid.

All experiments were successful. Figure 3(e-h) shows sample results from the 3 different environments. Landmarks were SIFT features [22] matched between current and goal images.

The high-resolution (3840 x 1024) Ladybug camera [25] captured images of a room (Figure 3(k)) and an office cubicle (l). Figure 3(e,f) are results for the room using conservative and non-conservative algorithms respectively. Room images were taken with the camera $0.85 \mathrm{~m}$ above the ground. This is necessary for the conservative method, which requires landmarks both above and below the image horizon. (In contrast, cubicle images were taken with the camera lying on the floor and were unsuitable for the conservative algorithm since everything below the horizon is featureless ground.)

Both conservative and non-conservative methods homed successfully in Figure 3(e,f). Since the conservative method discards many constraints, the homing direction deviated further from $\overrightarrow{C G}$, compared to the non-conservative method which gave more direct homing paths. Nevertheless, it is clear that a robot placed anywhere within the grid will follow the vectors and eventually reach the goal position.

Figure $3(\mathrm{~g})$ demonstrates successful homing in the cubicle environment using the non-conservative method. We also 


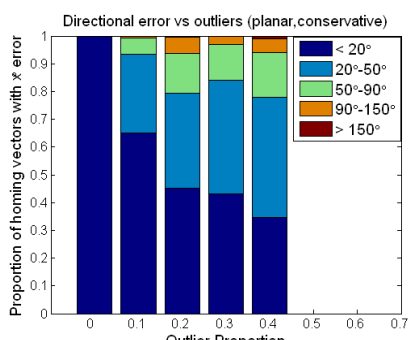

(a)

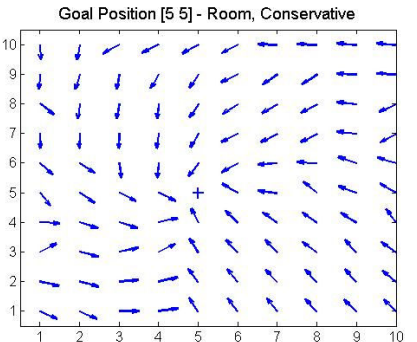

(e)

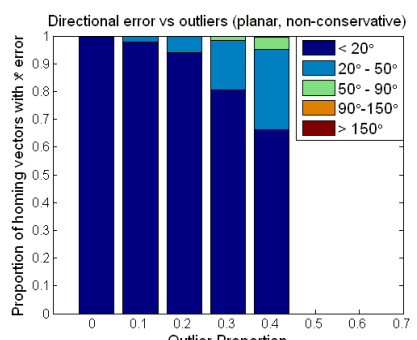

(b)

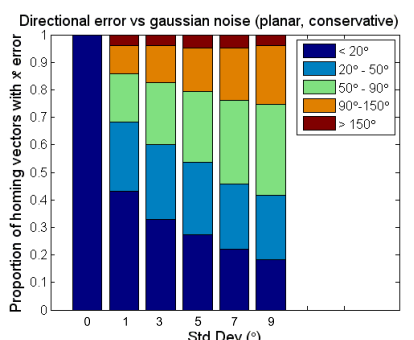

(c)

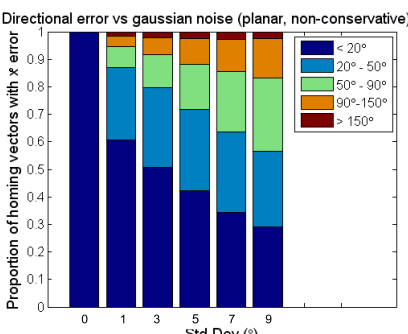

(d)

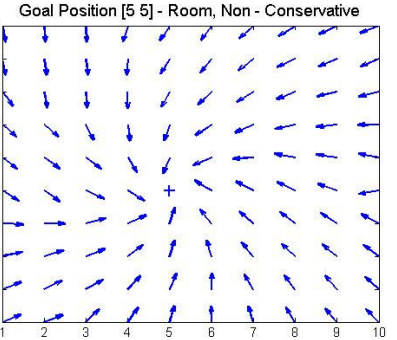

(f)

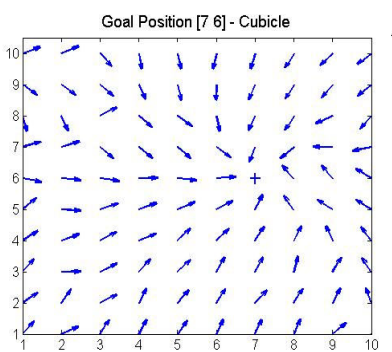

(g)

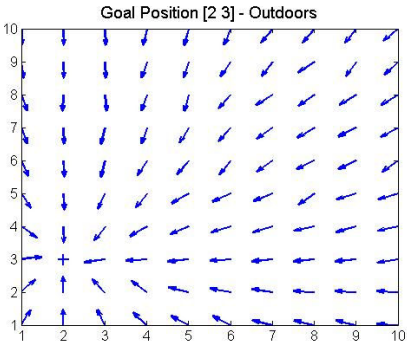

(h)

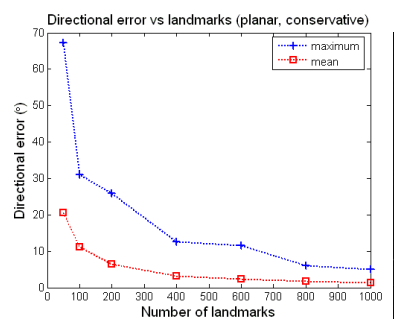

(i)

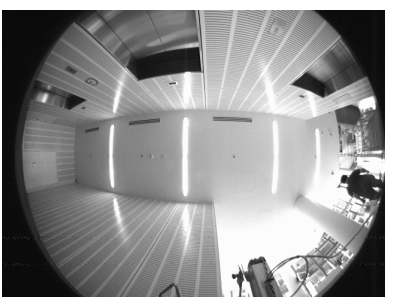

(j)

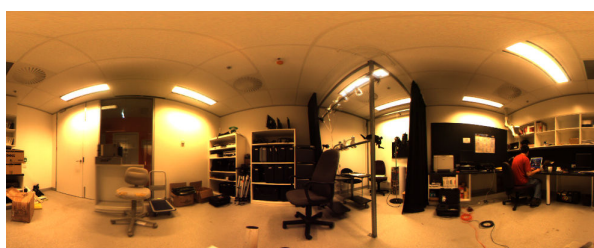

(k)

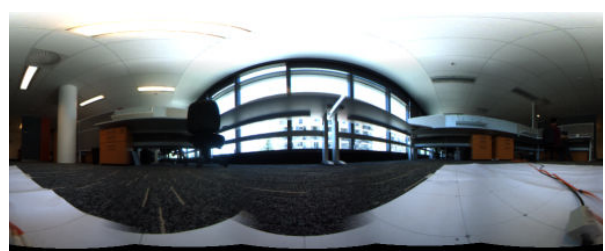

(1)

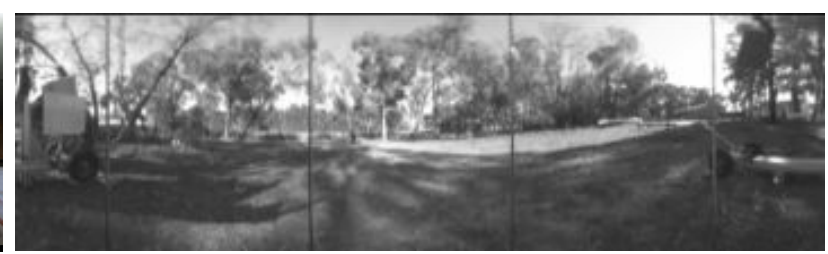

(m)

Fig. 3. (a-d) Homing direction under outliers and Gaussian noise. (e-f) Grid trials for room, comparing conservative and non-conservative methods. (g-h) Grid trials in cubicle and outdoors. (i) Effect of varying number of landmarks (j) Robot's view in atrium experiment. (k) High-resolution panorama taken in a room with an elevated camera and (l) taken in a cubicle with camera on the floor. (m) Low-resolution panorama taken outdoors.

tested the algorithm on a low-resolution $(360 \times 143)$ outdoor image set supplied by authors of $[17,21]$. Figure $3(\mathrm{~m})$ is an example image from the set and $3(\mathrm{~h})$ is a sample result. Interestingly, the low-resolution outdoor images gave smoother vector fields than the high-resolution, indoor ones. We believe this is due to a more even distribution of landmarks outdoors, whereas indoors, features tend to be denser in some areas of the image but are very sparse in other areas, leading to a bias in some directions. However, convergence is unaffected and homing paths remain fairly straight.

Robot trials: Videos at [26]. A holonomic wheeled robot [27] homed in various environments including an office and a building atrium. The robot performed local homing multiple times to move along a chain of goal positions (simplest instance of the topological maps mentioned in Section I) in order to get from one part of the environment to another. Snapshots were captured at intermediate goal positions and stored in the chain. An Omnitech Robotics fish-eye lens camera [28] was mounted pointing upwards, giving views such as Figure 3(j), where the image rim corresponds to the horizon.

Homing was successful to within the order of a few centimeters, in the presence of mismatched landmarks, moving objects (leading to bad landmark points) and illumination changes.

\section{DISCUSSION}

Robustness to outliers: In methods such as [11, 12, 10, 20], a series of vectors, $\left\{M_{1}, M_{2} \cdots M_{N}\right\}$, are derived from landmark bearings by some rule, and the average direction is taken as the homing vector, $M_{r e s}=M_{1}+M_{2}+\cdots+M_{N}$. Unfortunately, this average is sensitive to outliers which can 
potentially cause the homing vector to point in any random direction. The experiments demonstrated that our voting-based method was able to efficiently incorporate information from a set of landmarks while being resistant to significant numbers of mismatched and moved landmarks in the set.

Number of landmarks: More landmarks lead to more direct homing paths. However, Observation 1 suggests that homing is successful as long as homing directions are consistently less than $90^{\circ}$ from $\overrightarrow{C G}$. This condition is satisfied even with as few as 50 landmark matches (Figure 3(i)). In the experiments, images typically gave hundreds of SIFT matches.

Speed: Our algorithm runs in $35 \mathrm{msec}$ ( $28 \mathrm{fps}$ ) on a Linux OS, Pentium4, 3GHz, 512Mb RAM machine ( $\sim 200$ landmarks). The inputs to the algorithm were SIFT matches in the experiments, but these can take several seconds to compute. However, real-time SIFT implementations (for example, on a GPU) do exist. Alternatively, faster feature tracking methods such as KLT are also possible.

Poor Image Resolutions: The trials with low-resolution (360 x 143 pixels) outdoor images demonstrate that the algorithm works well even with poor image resolutions. This is in agreement with the results of the simulations under increasing Gaussian noise. The image noise in most cameras is generally in the order of a couple of pixels (typically less than a degree), which is far less than the amounts of Gaussian noise used in the simulations (up to $9^{\circ}$ ). Therefore, what these large noise trials do in fact simulate is the degradation of accuracy caused by using very coarse image resolutions causing uncertainty in the landmark ray.

Voting Resolution: Lower voting resolutions (currently $1^{\circ}$ per bin) could achieve even greater speeds without affecting convergence (it will, however, lead to a less direct path home). As an extreme example, the conditions of Observation 1 are satisfied even with as few as 4 voting bins ( $90^{\circ}$ per bin), if $\mathbf{~}$ is the center of the bin and conservative voting was used. Such a minimalist version of the method is well-suited for small, resource limited robots.

Flexible Probability Maps: Whilst our experiments (and most existing methods) obtained a single homing vector, the voting table is in fact a weighted map of likely directions for homing. This leads to a flexible and natural framework for incorporating additional navigational constraints such as obstacle avoidance, kinematic constraints (in non-holonomic robots), centering behaviour and path optimality planning. For example, directions blocked by obstacles can be augmented with negative votes so that the robot is discouraged from moving in that direction.

\section{CONCLUSION}

We investigated the problem of planar visual homing using landmark angles and presented three novel algorithms and demonstrated their performance in simulations and real robot trials. Videos available on the author's website [26].

Acknowledgments: We thank Jochen Zeil for providing the outdoor image set and Luke Cole for help with the robot.
NICTA is funded by the Australian Government as represented by the Department of Broadband, Communications and the Digital Economy and the ARC through the ICT Centre of Excellence program.

\section{REFERENCES}

[1] S. Thrun, W. Burgard, D. Fox, Probabilistic Robotics, MIT Press, 2005.

[2] A. Davison, Real-time simultaneous localisation and mapping with a single camera, Proc. International Conference on Computer Vision, 2003.

[3] R. Hartley and A. Zisserman, Multiple View Geometry in Computer Vision, Cambridge University Press, 2000.

[4] S. Hutchinson, G. Hager and P. Corke, A tutorial on visual servo control, IEEE Trans. Robotics and Automation, vol. 12, no. 5, pp. 651-670, 1996.

[5] D.T. Lawton and W. Carter, Qualitative spatial understanding and the control of mobile robots, IEEE Conf. on Decision and Control, vol. 3 , pp. 1493-1497, 1990.

[6] J. Hong, X. Tan, B. Pinette, R. Weiss and E.M. Riseman, Image-based homing, IEEE Control Systems Magazine, vol. 12, pp. 38-45, 1992.

[7] M.O. Franz, B. Schölkopf, H.A. Mallot, H.H. Bülthoff, Learning view graphs for robot navigation, Autonomous Robots, vol. 5, no. 1, 1998, pp. 111-125.

[8] M.O. Franz, H.A. Mallot, Biomimetic robot navigation, Biomimetic Robots, Robotics and Autonomous Systems, vol. 30 no. 1, 2000, pp. 133-153.

[9] B. Kuipers and Y.T. Byun, A robot exploration and mapping strategy based on a semantic hierarchy of spatial representations, Robotics and Autonomous Systems, vol. 8 no. 12, 1991, pp. 47-63.

[10] K.E. Bekris, A.A. Argyros, L.E. Kavraki, Angle-Based Methods for Mobile Robot Navigation: Reaching the Entire Plane, in Proc. Int. Conf. on Robotics and Automation, pp. 2373-2378, 2004

[11] D. Lambrinos, R. Möller, T. Labhart, R. Pfeifer and R. Wehner, A mobile robot employing insect strategies for navigation, Biomimetic Robots, Robotics and Autonomous Systems, vol. 30, no. 1, 2000, pp. 39-64.

[12] S.G. Loizou and V. Kumar, Biologically Inspired Bearing-Only Navigation and Tracking, IEEE Conf. on Decision and Control, 2007.

[13] K. Weber, S. Venkatesh and M. Srinivasan, Insect-inspired robotic homing, Adaptive Behavior, vol. 7, no. 1, 1999, pp. 65-97.

[14] S. Gourichon, J.A. Meyer and P. Pirim, Using colored snapshots for short range guidance in mobile robots, Int. J. Robot. Automation, vol. 17, pp. 154-162, 2002.

[15] M.O. Franz, B. Schölkopf, H.A. Mallot, H.H. Bülthoff, Where did I take that snapshot? Scene-based homing by image matching, Biological Cybernetics, vol. 79, no. 3, 1998, pp. 191-202.

[16] B.A. Cartwright and T.S. Collett, Landmark learning in bees, Journal of Comparative Physiology A, vol. 151, no. 4, 1983, 521-543.

[17] J. Zeil, M.I. Hoffmann and J.S. Chahl, Catchment areas of panoramic images in outdoor scenes, Journal of the Optical Society of America A, vol. 20 , no. 3,2003 , pp. 450-469.

[18] A. Vardy, R. Möller, Biologically plausible visual homing methods based on optical flow techniques, Connection Science, vol. 17, no. 1, 2005, pp. 47-89.

[19] R. Möller, Insect visual homing strategies in a robot with analog processing, Biological Cybernetics: special issue in Navigation in Biological and Artificial Systems, vol. 83, no. 3 pp. 231-243, 2000.

[20] A.A. Argyros, C. Bekris, S.C. Orphanoudakis, L.E. Kavraki, Robot Homing by Exploiting Panoramic Vision, Journal of Autonomous Robots, Springer, vol. 19, no. 1, pp. 7-25, July 2005.

[21] W. Stürzl, J. Zeil, Depth, contrast and view-based homing in outdoor scenes, Biological Cybernetics, vol. 96, pp. 519-531, 2007.

[22] D.G. Lowe, Object Recognition from local scale-invariant features, in Proc. of Int. Conf. on Computer Vision, pp. 1150-1157, September, 1999.

[23] C. Harris and M. Stephens, A combined corner and edge detector, Proceedings of the 4th Alvey Vision Conference, pp. 147-151, 1988.

[24] J. Shi and C. Tomasi, Good Features to Track, IEEE Conference on Computer Vision and Pattern Recognition, pp. 593-600, 1994.

[25] Point Grey Research, http://www.ptgrey.com , 2009.

[26] J.Lim, http://users.rsise.anu.edu.au/\%7Ejohnlim/ , 2009.

[27] L. Cole and N. Barnes, Insect Inspired Three Dimensional Centering, In Proc. Australasian Conf. on Robotics and Automation, 2008.

[28] Omnitech Robotics, http://www.omnitech.com/ , 2009. 